\title{
Los Acuerdos de Asociación de la UE con África y América Latina: La nueva apuesta europea por Tratados de Libre Comercio con cooperación
}

\author{
EDUARDO BIDAURRATZAGA \\ Instituto Hegoa, UNIVERSIDAD DEL PAÍS VASCO (UPV/EHU), ESPAÑA. E-mail: \\ eduardo.bidaurratzaga@ehu.es
}

PATXI ZABALO

Instituto Hegoa, UNIVERSIDAD DEL PAÍS VASCO (UPV/EHU), ESPAÑA. E-mail: patxi.zabalo@ehu.es

\section{RESUMEN}

Este trabajo estudia el alcance y los potenciales efectos de los Acuerdos de Asociación impulsados por la Unión Europea para institucionalizar sus relaciones con países o grupos de países africanos y latinoamericanos. El contenido de los acuerdos va más allá de los tradicionales aspectos comerciales en dos sentidos. Por un lado, porque, aunque incluyen una zona de libre comercio, también tienen una componente política y otra de cooperación para el desarro1lo. Por otro, porque los acuerdos abordan un conjunto de asuntos que pretenden ir más allá de los cubiertos por la OMC.

Palabras clave: Acuerdos de Asociación, Unión Europea, África, América Latina, desarrollo.

\section{European Partnership Agreements with Africa and Latin America: The EU's New Free Trade with Aid Formula}

\begin{abstract}
This paper examines the scope and potential effects of the association agreements promoted by the European Union in order to institutionalize its relations with countries or groups of countries in Africa and Latin America. The content of the agreements goes beyond traditional trade issues in two ways. On the one hand, apart from a free trade area, they also include political and development cooperation components. On the other, the agreements address a set of issues that aim to go beyond those covered by the WTO.
\end{abstract}

Keywords: Association/Partnership Agreements, European Union, Africa, Latin America, Development.

Clasificación JEL: F55, F13, F23, F15

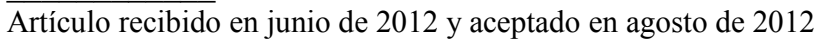

Artículo disponible en versión electrónica en la página www.revista-eea.net, ref. ə-30305 


\section{INTRODUCCIÓN}

Las relaciones entre la Unión Europea (UE) y dos de las regiones con las que históricamente varios de sus países miembros han mantenido unas relaciones preferentes, África y América Latina, han sufrido importantes transformaciones en los últimos tiempos. Aunque con características específicas para cada una de ellas en función del origen y la evolución de dichas relaciones, el núcleo del modelo ahora reconfigurado es común para ambas: la apuesta por la reciprocidad comercial a partir de la creación áreas de libre comercio, la inclusión de una agenda de negociación de temas que van más allá del marco multilateral de la OMC, y la combinación de todo ello con una política específica de la UE en materia de cooperación al desarrollo, a la que se le suma el componente de diálogo político.

El presente trabajo revisa la bibliografía, los acontecimientos más recientes y algunas estadísticas sobre esta problemática con el objetivo de analizar las transformaciones producidas y los principales argumentos suscitados en el debate sobre los pros y contras de estos acuerdos. La pregunta que se plantea es si los Acuerdos de Asociación impulsados por la UE contribuyen a mejorar o deteriorar las condiciones para la promoción del desarrollo en los países o grupos de países en ambas regiones. Para ello, se realiza inicialmente un repaso de los antecedentes y la evolución de las relaciones entre la UE y cada región. A continuación se analizan los principales cambios producidos y los temas incluidos en la agenda de negociación. Finalmente se estudian los argumentos justificadores de dichos cambios y se examinan los diversos efectos potenciales de estos nuevos acuerdos sobre las economías africanas y latinoamericanas en términos de mejoras o deterioros de sus condiciones de desarrollo.

\section{ORIGEN, EVOLUCIÓN Y CONTENIDO DE LOS ACUERDOS}

Desde que se constituyó la Comunidad Económica Europea (CEE) en 1958 con seis países miembros, la política comercial y de cooperación comunitaria ha tenido un tratamiento específico para las excolonias europeas de África Subsahariana ${ }^{1}$, estructurada posteriormente, desde 1975, en el marco de los Acuerdos de Lomé. En cambio, América Latina no ha sido objeto de especial atención comunitaria hasta mediados de los años 1990, al lanzarse la idea de celebrar acuerdos de asociación con diversos países y grupos de países del Sur económico, reforzada en 2006 con la estrategia Europa Global (Comisión Europea, 2006).

Estos antecedentes han tenido una clara influencia sobre el destino de los flujos de AOD de este grupo de actores relevantes de la comunidad donante

\footnotetext{
${ }^{1}$ Los cinco países del Norte de África quedaron encuadrados en la política mediterránea de la CEE y por tanto fuera del marco de relaciones del grupo de países ACP.
} 
formado por las instituciones de la UE y sus países miembros. De hecho, tal y como se observa en la Figura 1, en la comparativa entre las dos regiones objeto de análisis, los flujos de AOD de las instituciones de la Unión Europea destinados a África Subsahariana son muy superiores a los recibidos por Latinoamérica y el Caribe. Sin embargo, la distribución regional de la AOD bilateral de sus miembros varía sustancialmente: España destina a Latinoamérica y el Caribe cantidades muy superiores al resto de sus vecinos, mientras el Reino Unido, segundo donante bilateral de la UE a África Subsahariana tras Francia, destina cantidades mínimas a Latinoamérica y el Caribe.

Figura 1

Reparto regional de la AOD de la Unión Europea y sus países miembros, promedio 2009-10

(millones de dólares)

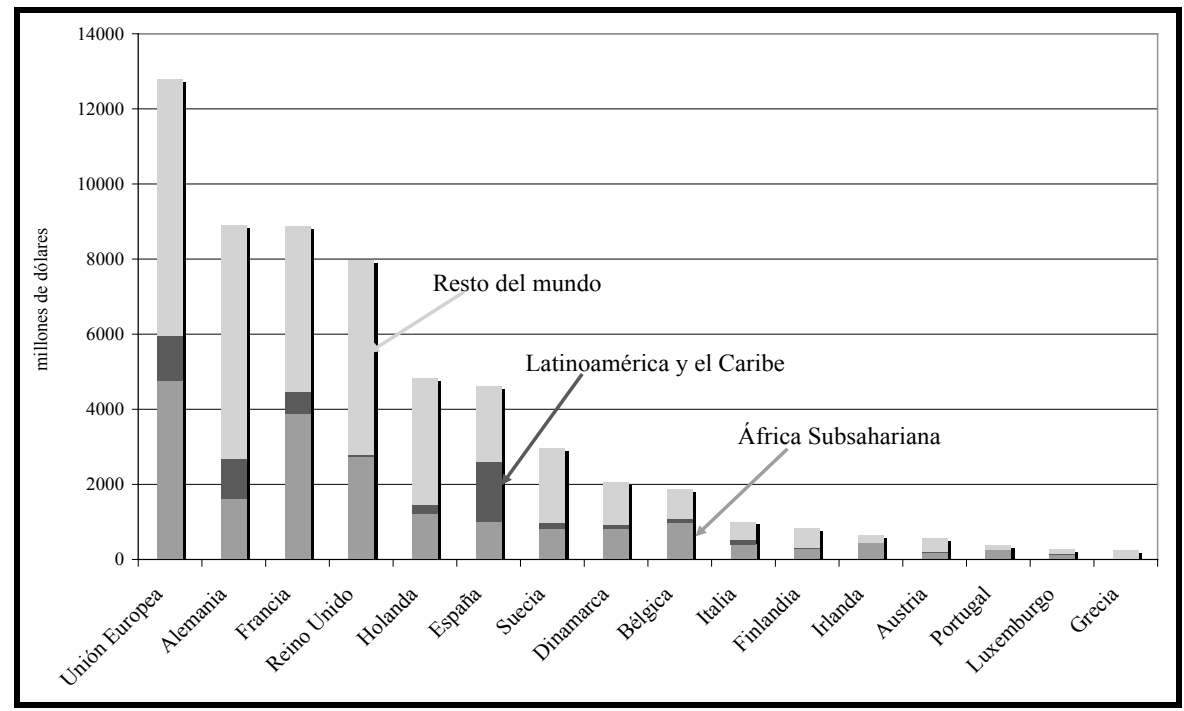

Fuente: Elaborado con datos del CAD (www.oecd.org/dac/stats).

\section{1. África Subsahariana}

El Fondo Europeo de Desarrollo (FED), creado por el Tratado de Roma como principal instrumento de cooperación al desarrollo de la CEE con sus antiguas colonias de África Subsahariana, estableció una relación privilegiada entre ambas partes, primero a través del Convenio de Yaundé I (1963-69), firmado por la CEE con 18 ex colonias, y seguidamente con Yaundé II (1969-75), que con la incorporación de Kenia, Tanzania y Uganda suscribieron 21 países africanos. Al ingresar el Reino Unido en la CEE, llegaron los sucesivos convenios de Lomé, rubricados por un número creciente de excolonias, que pasaron 
de ser 45 en Lomé I (1975-79) a 71 de Lomé IV (1990-2000). Para coordinar sus actividades estos países constituyeron la asociación de estados de África, Caribe y Pacífico (ACP), que cuenta hoy en día con 79 países. Y desde 2000 las relaciones entre la Unión Europea y los países ACP se rigen por el Acuerdo de Cotonú, dando fin a la denominada era Lomé.

El modelo de Lomé ha sido un elemento central de la política de cooperación al desarrollo de la CEE/UE. Además de asistencia técnica y financiera de la mano de consecutivos FED (IV-VIII), tomaba como una de sus señas de identidad el acceso preferencial no recíproco al mercado europeo de los productos procedentes de los países ACP. En un contexto de reclamaciones a favor de un Nuevo Orden Económico Internacional (NOEI), esta discriminación positiva sin contrapartida se presentaba como una contribución europea al desarrollo socioeconómico de los países ACP. Sin embargo, resultaba difícil ocultar que estas relaciones proporcionaban a la CEE un acceso privilegiado a las materias primas de sus excolonias en un momento de subida del precio de estos bienes primarios en los mercados internacionales. Además, este régimen preferencial ha contado con la destacada excepción de los productos protegidos por la Política Agraria Común (PAC) europea. De este modo, en el sector agrícola son básicamente los productos tropicales los que han gozado de condiciones de acceso más favorables al mercado europeo.

Cuando Lomé VI se acercaba a su expiración, a finales de 1998 se puso en marcha el proceso de negociación del que surgió el Acuerdo de Cotonú. En esas negociaciones la Unión Europea presionó fuertemente para reorientar el marco de relaciones previo hacia una liberalización comercial reciproca. Sin embargo, los representantes de los países ACP apostaban en todo momento por la prorroga del modelo anterior durante el mayor tiempo posible y, en su defecto, por la flexibilidad de los nuevos acuerdos en cuanto a temas sensibles y periodos de transición que permitieran un ajuste no traumático a la nueva situación (Hurt, 2003). Tras año y medio de negociaciones, en junio de 2000 se firmó el Acuerdo de Cotonú, que sustituye al marco regulador de los 25 años anteriores, pero prolonga sus preferencias de forma transitoria hasta el cierre de las negociaciones sobre los Acuerdos de Asociación Económica (AAE, EPA por sus siglas en inglés), previsto para antes de finalizar el $2007^{2}$.

Aunque en el ámbito político el acuerdo incluye un amplio abanico de temas novedosos y el refuerzo de algunos ya existentes, en Lomé IV revisado (derechos humanos, democracia, gobernanza...) las transformaciones más significativas se producen en su componente económico y particularmente en el comercial. En efecto, el cambio fundamental es la sustitución del antiguo régi-

\footnotetext{
${ }^{2}$ El modelo de Lomé se siguió aplicando al aceptar la Organización Mundial de Comercio las dos exenciones temporales solicitadas por la UE, que expiraron el 31-12-2007.
} 
men comercial de preferencias unilaterales por otro de carácter recíproco, de tal forma que la liberalización comercial se produzca en ambas direcciones, mediante el establecimiento de áreas de libre comercio ${ }^{3}$. A pesar de todo, este nuevo modelo europeo pretende diferenciarse del estadounidense, evitando la expresión libre comercio, con el objeto de enfatizar la idea de que los Acuerdos de Asociación Económica, además de un régimen comercial recíproco, incorporan también asistencia técnica y financiera. Y lo hacen en amplias áreas como desarrollo económico, desarrollo social y humano, y cooperación regional e integración por medio de los FED IX y X para los periodos 2000-07 y 2008-13 respectivamente ${ }^{4}$. Además se mantiene el anterior régimen preferencial no recíproco para los Países Menos Avanzados (PMA), con independencia de si forman parte del grupo ACP o no, gracias a la iniciativa Todo Menos Armas ${ }^{5}$ lanzada por la UE en 2001.

En su condición de acuerdo para acordar, el Acuerdo de Cotonú no define con detalle los contenidos de cada AAE. Se limita a indicar que deberían negociarse preferentemente con grupos regionales preestablecidos, para su puesta en marcha a comienzos del 2008, mediante la progresiva liberalización comercial y su definitiva entrada en vigor no más tarde del año 2020. Así, tras un año de negociaciones conjuntas, en septiembre del 2003 comenzó el proceso de negociación entre la Unión Europea y algunos grupos regionales, si bien otros no empezarían hasta el año siguiente (Marín, 2008).

De este modo, se han establecido siete marcos de negociación independientes en el interior del grupo ACP, en gran medida agrupados sobre la base de los acuerdos de integración económica existentes. Cinco de ellos están formados por países africanos, configurando la siguiente subdivisión: 1) la Comunidad Económica de Estados de África del Oeste (ECOWAS-CEDEAO) más otro país de la región; 2) la Comunidad Económica y Monetaria de África Central (CEMAC) y otros dos países de la región; 3) ocho de los catorce miembros de la

${ }^{3}$ Dos instrumentos de ayuda, como Stabex y Sysmin, creados con el objetivo de estabilizar los ingresos provenientes de las exportaciones de productos agrícolas y mineros respectivamente en el marco de Lomé, fueron sustituidos en el Acuerdo de Cotonú por el denominado FLEX (Fluctuations in Export Earnings).

${ }^{4}$ El décimo FED prevé para el periodo 2008-13 una dotación de 22.682 millones de euros, de los que el $97 \%$ va destinado a los países ACP. De este total el $81 \%$ se destina a programas nacionales o regionales, el $12 \%$ a cooperación intrarregional y el $7 \%$ a la promoción de inversiones. Asimismo se incluye a modo de novedad el uso de recursos extra a modo de incentivos en el ámbito político para países que mejoren su gobernanza en aspectos como la gestión financiera, impositiva o de los marcos legales (European Union, 2006).

${ }^{5}$ La iniciativa Todo Menos Armas permite a los PMA acceso al mercado europeo libre de aranceles y cuotas para todos los productos, con la excepción de armas. Además de este caso, explícitamente mencionado, esta iniciativa excluye de forma transitoria ciertos productos agrícolas, como el plátano, el arroz y el azúcar (Marín, 2008). De los 49 Países Menos Avanzados reconocidos por la UNCTAD, 39 son estados ACP, la mayoría africanos (36). 
Comunidad de Desarrollo de África Austral (SADC); 4) el conocido como grupo ESA (grupo de negociación para los acuerdos, pero no un grupo de integración propiamente dicho), formado por once países del Este y Sur de África, varios de los cuales son miembros del Mercado Común para África Oriental y Austral (COMESA) o de la SADC; 5) los cinco miembros de la Comunidad de África del Este (EAC), escindidos en 2007 del grupo ESA. Los otros dos marcos de negociación corresponden a: 6) catorce islas-estado del Pacífico; y 7) los quince países del Caribe, agrupados en el CARIFORUM (Comisión Europea, 2012).

La negociación de los AAE ha enfrentado planteamientos sustancialmente diferentes respecto a dos temas: el calendario para la conclusión de las negociaciones y la puesta en marcha de los acuerdos, y el propio alcance de dichos acuerdos. Desde el comienzo del proceso de negociación, la Comisión Europea ha trabajado de forma inflexible con el horizonte temporal de puesta en marcha de los AAE en el primer día de enero de 2008. Su argumentación principal se ha basado en la expiración de la prórroga concedida por la Organización Mundial de Comercio (OMC) sobre la exención que ha permitido durante los últimos años aplicar las preferencias no recíprocas de Lomé, incompatibles con su normativa comercial multilateral. En este sentido, la UE ha dado a entender en todo momento que en ausencia de acuerdo no quedaría otra posibilidad que el tratamiento Todo Menos Armas para los PMA y la degradación al unilateral Sistema de Preferencias Generalizadas (SPG) para el resto.

Contrariamente a esta posición, la mayor parte de los gobiernos de los países africanos y diversas redes de movimientos sociales y organizaciones no gubernamentales europeas y africanas ${ }^{6}$ han insistido en la necesidad de flexibilizar los plazos y restringir los temas incluidos en la agenda de negociación, apostando por las búsqueda de alternativas a los AAE, tal y como el propio Acuerdo de Cotonú recomienda en ausencia de acuerdo. Sus propuestas han ido desde la inclusión de temas relativos exclusivamente al comercio de mercancías (EPA light) o la solicitud de una nueva prorroga de la exención correspondiente en la OMC que permita seguir aplicando Lomé hasta la concesión por parte de la UE del denominado $S P G+$ o mejorado ${ }^{7}$. Todo ello pone de manifiesto un problema de falta de voluntad política más que de posibilidad real de ofertar alternativas apropiadas para mejorar las condiciones de los acuerdos para las economías del grupo ACP (Mold, 2007; Marín, 2008).

\footnotetext{
${ }^{6}$ Ver campaña "Stop EPAs", lanzada en el Foro Social Africano de 2004.

${ }^{7}$ Mediante el denominado SPG+, la UE proporciona mayores preferencias arancelarias que el SPG a los países en desarrollo con economías más débiles que acepten una serie de acuerdos internacionales sobre derechos humanos, sostenibilidad y buen gobierno, entre otros.
} 
Como resultado, en enero de 2008 solamente había un AAE total, el de los países del Caribe. El resto de países ACP, bien sea de forma individual o en grupo, solo habían establecido acuerdos interinos o parciales. En los cinco grupos de países africanos señalados, la situación actual de las negociaciones de AAE interinos es la siguiente:

En África Occidental no se ha alcanzado ningún acuerdo conjunto a nivel regional. Sin embargo, dos países de forma individual, Costa de Marfil y Ghana, suscribieron sus propios AAEs interinos en diciembre de 2007. Nigeria, el otro no-PMA de la región, no ha firmado ningún acuerdo interino, y ha solicitado ser incluido en el formato SPG+, estatus que le ha sido denegado, quedando así relegado al régimen SPG estándar. Finalmente, Cabo Verde, que perdió su estatus de PMA en enero del 2008, goza de una prorroga de tres años para poder exportar en función del régimen Todo Menos Armas correspondiente a los PMA.

En África Central tampoco ha habido ningún acuerdo interino regional, si bien uno de los no-PMA, Camerún, obtuvo uno de carácter individual en diciembre de 2007, finalmente firmado en enero de 2009, que está encontrando serias resistencias para su puesta en práctica. Los otros dos no-PMA de la región, Gabón y República del Congo, siguen sin contar con ningún acuerdo interino individual y exportando, por tanto, bajo el régimen SPG estándar desde enero de $2008^{8}$.

En África Austral, identificada como región SADC, se ha alcanzado un acuerdo regional interino por parte de una pequeña minoría de sus miembros (tan sólo cinco: Botswana, Lesotho, Swazilandia, Mozambique y Namibia), que incluye tanto PMA como no-PMA. Sin embargo, Namibia sigue sin ratificar el acuerdo interino (firmado por el resto en 2009), lo que está retrasando considerablemente su puesta en marcha. Los principales obstáculos esgrimidos por los representantes namibios tienen que ver con la amenaza que el AAE supone para el proceso de integración regional, en referencia directa a la $\mathrm{SACU}^{9}$, así como con las posibilidades de desarrollo de sus propias industrias.

Del grupo ESA, tan solo seis países (Seychelles, Zimbabwe, Mauricio, Comoros, Madagascar y Zambia) habían alcanzado un acuerdo interino para finales de 2007, firmado en 2009 por todos menos por Comoros y Zimbabwe. El acuerdo incluye asimismo tanto países menos avanzados como no-PMA. Los

\footnotetext{
${ }^{8}$ Gabón solicitó ser incluido en la lista de países beneficiados por el régimen $\mathrm{SPG}+$, pero fue rechazado por no haber ratificado todas las convenciones internacionales requeridas.

${ }^{9}$ La Unión Aduanera de África Austral (SACU) está formada por Botsuana, Lesoto, Suazilandia, Namibia, y Sudáfrica. En los últimos años, como consecuencia de la firma de AAE interinas con la UE por los tres primeros miembros, los otros dos han hecho llamamientos a la negociación conjunta por parte de toda la unión aduanera, tal y como sus propias normas internas establecen.
} 
países miembros de EAC (Kenia, Tanzania, Uganda, Burundi y Ruanda) decidieron en noviembre de 2007 suscribir un AAE interino al margen del resto de países de África Oriental y Austral. En estos dos acuerdos también participan PMA junto a un no-PMA, Kenia.

De este modo, en estos grupos regionales la mayoría de los PMA se ha acogido a la prolongación del régimen comercial preferencial no recíproco por medio de la iniciativa Todo Menos Armas. Por su parte, la mayoría de los no-PMA africanos (18) ha firmado algún tipo de acuerdo parcial, bien de forma individual o dentro de un grupo, aunque con calendarios de liberalización diferentes. La excepción a esta regla la constituyen los casos de Nigeria, República del Congo, Gabón y Sudáfrica, si bien este último ya cuenta previamente con su propio acuerdo comercial individual con la Unión Europea (Trade Development and Cooperation Agreement, TDCA) (ECDPM, 2011; Comisión Europea, 2012; Marín, 2008).

Como consecuencia de todo ello, por la vía de los acuerdos interinos se ha solucionado el problema de incompatibilidad con la normativa de la OMC. No obstante, estos acuerdos no incluyen otro tipo de temas más sensibles y polémicos, como son el comercio de servicios y los temas relacionados con el comercio: liberalización de inversiones, contrataciones públicas, y derechos de propiedad intelectual, entre otros.

En cualquier caso, es importante recordar que estos acuerdos incluyen una cláusula de revisión (rendez-vous) de tal forma que se asume que el proceso de negociación no se da por finalizado y se supone que los países ACP tienen que seguir negociando sobre los citados temas en el futuro. Todo ello genera amplia controversia entre las partes, pues mientras los países africanos han manifestado su posición contraria a la inclusión de estos temas sensibles, la Unión Europea se aferra a su postura, mezcla de interesada y paternalista, y sigue insistiendo en las grandes ventajas de todo ello para esos países de cara a su apropiada inserción en la economía mundial (Bidaurratzaga, 2008).

Téngase en cuenta a este respecto que durante la última década la balanza comercial de la UE con África ha sido deficitaria hasta el año 2009 en que pasa abruptamente a ser ligeramente superavitaria como consecuencia del descenso de la importaciones por la crisis, en un contexto en el que el peso de la UE en el comercio con el continente ha sido decreciente. (EUROSTAT, 2010). Esto, junto con el posicionamiento creciente de otras economías emergentes en el continente africano, ayuda a entender el empeño por parte de la UE de atar formalmente su área de influencia mediante acuerdos comerciales recíprocos de los que pueda beneficiarse.

\subsection{América Latina y el Caribe}

Sobre la base de unas relaciones previas diferentes, pero con unos tiempos 
similares a los recién mencionados para los países ACP, la Unión Europea comenzó a promover la consecución de Acuerdos de Asociación (AdA) con países latinoamericanos desde mediados de los años 1990. Esto suponía una modificación de su política previa, que se limitaba a aplicar las preferencias unilaterales concedidas al conjunto de economías del Sur que no eran países ACP, esencialmente mediante el régimen del SPG. Este cambio se explica en gran medida por la necesidad de adaptarse a las normas de la OMC, en vigor desde 1995, que restringen el uso del trato preferencial no recíproco a los PMA, categoría a la que solo pertenece Haití en la región. A su vez, el lento avance de las negociaciones de la Ronda de Doha de la OMC también representa un estímulo para tratar de conseguir por la vía bilateral lo que no se obtiene en la multilateral. Además, como indica la estrategia Europa Global, la UE pretende competir de manera más activa con Estados Unidos en los mercados globales, incluida su propia área de influencia latinoamericana, al igual que con China (Comisión Europea, 2006).

En estas circunstancias, la Unión Europea y América Latina y el Caribe acordaron en la Cumbre de Río de Janeiro de 1999 desarrollar una asociación estratégica birregional para impulsar sus relaciones políticas, económicas y culturales. Estas cumbres se han venido repitiendo cada dos años, hasta la de Madrid en mayo de 2010, estando previsto celebrar la próxima reunión en Santiago de Chile en 2012 (Comisión Europea, 2005 y 2009; Consejo de la UE, 2010).

Así, en los últimos años la UE ha suscrito diversos acuerdos de asociación, que frente al impulso por parte de Estados Unidos de meros tratados de libre comercio (TLC), además del componente comercial, incluye otros dos temas: cooperación y diálogo político. Por lo que respecta al primero, más allá de los discursos y las declaraciones de principios, desde posiciones críticas con estos acuerdos de la UE se ha planteado que este pilar de cooperación supone un aspecto secundario frente al económico y el comercial en particular (Morales y Garza, 2009). Y en cuanto al diálogo político, los valores promocionados por la UE a partir de los años noventa (libertades democráticas, derechos humanos, buen gobierno...), luego formalizados en el Consenso Europeo de Desarrollo de 2005, han encontrado con frecuencia oposición por parte de algunos gobiernos en temas sobre gobernanza o respeto de derechos humanos y han abierto controversias también a la hora de tratar temas como el migratorio en términos de seguridad o de promoción del desarrollo humano y la cohesión social (Mata y Cordero, 2009; Morales y Garza, 2009).

$\mathrm{Al}$ igual que ocurre con los $\mathrm{AAE}$, los tratados de libre comercio incluidos en los AdA promueven la liberalización del comercio de mercancías, aunque para los productos agrícolas sensibles existen notables excepciones. También incluyen temas como la liberalización del comercio de servicios y la protección de la 
propiedad intelectual y contienen capítulos específicos sobre contratación pública, política de competencia e inversión. Y a la amplitud de asuntos abarcados unen su carácter expansivo, a través de cláusulas progresivas o de revisión, para garantizar que el proceso de negociación sobre estos temas sensibles continúe en el futuro (Lara y Silva, 2009; Caballeros, 2008; Kucharz y Vargas, 2010).

El primer acuerdo se firmó en 1997 con México y entró plenamente en vigor en 2000. El segundo AdA de la Unión Europea en América Latina se rubricó con Chile en 2002, entrando en vigor sus aspectos comerciales en 2003 y el resto en 2005. En mayo de 2010, durante la VI Cumbre UE-ALC, se dieron por concluidas las negociaciones de los AdAs de la Unión Europea con Centroamérica, por un lado, y con dos países andinos, Colombia y Perú, por otro. En 2011 se firmaron ambos AdA, que solo están pendientes de su ratificación para entrar en vigor (Comisión Europea, 2012).

Las negociaciones que han dado lugar al AdA entre la UE y Colombia y Perú, comenzaron con el conjunto de la Comunidad Andina, pero Bolivia las abandonó en 2008, en desacuerdo por el tratamiento otorgado a ciertos aspectos como a la propiedad intelectual y la biodiversidad. En julio de 2009 también Ecuador se retiró de las negociaciones por diferencias respecto a los derechos de los inmigrantes, los acuerdos sobre inversiones y el conflicto del banano, pero las retomó en febrero de 2010 al mejorar las condiciones ofrecidas por la UE para ese producto (INTAL, 2010). Por ello, no puede descartarse que termine sumándose al AdA, aunque sigue siendo motivo de fuerte controversia política en el interior del país.

El AdA entre la UE y Centroamérica incluye a los cinco países del Mercado Común Centroamericano (Costa Rica, El Salvador, Guatemala, Honduras y Nicaragua) y Panamá. Este es, de momento, el único AdA suscrito entre la UE y un bloque subregional, a pesar de que el mandato comunitario habla de fortalecer la integración regional latinoamericana, avanzando mediante acuerdos región a región. De hecho, antes de que empezaran las negociaciones en 2006, la UE exigió como condición previa ineludible una profundización en el proceso de integración centroamericano que, entre otras cosas, garantizase la libre circulación dentro de la región de las mercancías provenientes de Europa. Esto debería suponer la culminación de una auténtica unión aduanera, la firma de un tratado sobre inversiones y comercio de servicios, y el establecimiento de un mecanismo jurídico que asegure la aplicación de la legislación económica regional en toda la zona. Y además incluye a Panamá, que para poder suscribir el AdA ha tenido que asumir todos los compromisos adoptados por los otros cinco países (Ocampo y Lizano, 2009; Granados, 2009).

Las negociaciones con el MERCOSUR comenzaron en el año 2000 y se suspendieron en 2004 por la negativa de la UE a ampliar el acceso de los productos agrícolas sudamericanos al mercado europeo y eliminar los subsidios a la agri- 
cultura comunitaria. Sin embargo, en 2010 se reactivaron a pesar de que las dificultades persisten. Y entre ellas no es la menor el lento avance de la integración regional del MERCOSUR, que ahora además incluye a Venezuela, ya que también en este caso la construcción de un verdadero mercado común es una exigencia de la UE (Lara y Silva, 2009).

Por otra parte, como ya se ha visto, la UE ha alcanzado un Acuerdo de Asociación Económica en el marco del Acuerdo de Cotonú con el CARIFORUM, que agrupa a lo miembros del CARICOM y la República Dominicana. Este AAE fue rubricado en 2008, salvo por Haití que lo hizo en 2009. Y, aunque no ha entrado plenamente en vigor, se aplica provisionalmente desde diciembre de 2008.

En definitiva, la política de la Unión Europea hacia África Subsahariana y América Latina a comienzos del siglo XXI solo mantiene algunas trazas de trato especial y diferenciado para los PMA. Para el resto la única propuesta es la reciprocidad comercial, a la que se suman otros temas sensibles más allá de los estrictamente comerciales. Si bien todo ello viene acompañado de un paquete más amplio en materia de cooperación al desarrollo y diálogo político, estos pasan a ser temas menores frente a la prioridad establecida sobre la promoción de tratados de libre comercio.

\section{LA AGENDA OMC-PLUS CON COOPERACIÓN}

Como ya se ha señalado, la Unión Europea pretende diferenciar sus acuerdos de asociación de los tratados de libre comercio de otros actores como Estados Unidos, incluyendo siempre en el paquete el ámbito de la cooperación, del que hace seña de identidad. Esto es consecuente con el peso e influencia de la UE en la comunidad donante en términos de montos totales de Ayuda Oficial para el Desarrollo (AOD), ya que la suma de la AOD bilateral de los países miembros y la multilateral de las instituciones comunes de la UE más que duplica la de Estados Unidos, primer donante mundial ${ }^{10}$.

De hecho, como miembros destacados de la comunidad donante, la UE y sus estados miembros han desempeñado un papel muy activo en la reformulación de la agenda de la cooperación durante los últimos años. En este sentido, la búsqueda de una mayor efectividad en las políticas de cooperación ha llevado a la UE hacia la puesta en práctica de los principios de la Declaración de París y del Plan de Acción de Accra, el uso de nuevos instrumentos (apoyo sectorial y presupuestario) y la preocupación por la coherencia de políticas, entre otros aspectos. Pero más allá del apoyo a las líneas generales de la agenda de la co-

${ }^{10}$ Según los datos del CAD, en el promedio de 2009 y 2010, la AOD estadounidense ascendió a 26.689 millones de dólares, mientras que la de las instituciones de la UE fue de 12.796 millones y la del conjunto de los países miembros sumaba 46.013 millones de dólares (CAD, 2012). 
operación actual, la UE entiende que, por coherencia con su visión del desarrollo y de la lucha contra la pobreza, es fundamental hacer hincapié en los programas relacionados con la promoción del comercio exterior en general y de la integración regional en particular, entre otros instrumentos, mediante fondos de ayuda para el comercio (Aid for Trade). Y el hecho de que la propia cooperación al desarrollo vaya también vinculada a la de promoción del comercio internacional es representativo de la centralidad de este pilar económico en el marco de los acuerdos de asociación.

Figura 2

Porcentaje de la AOD* de las instituciones de la Unión Europea, sus países miembros y Estados Unidos recibido por África Subsahariana y Latinoamérica y el Carible

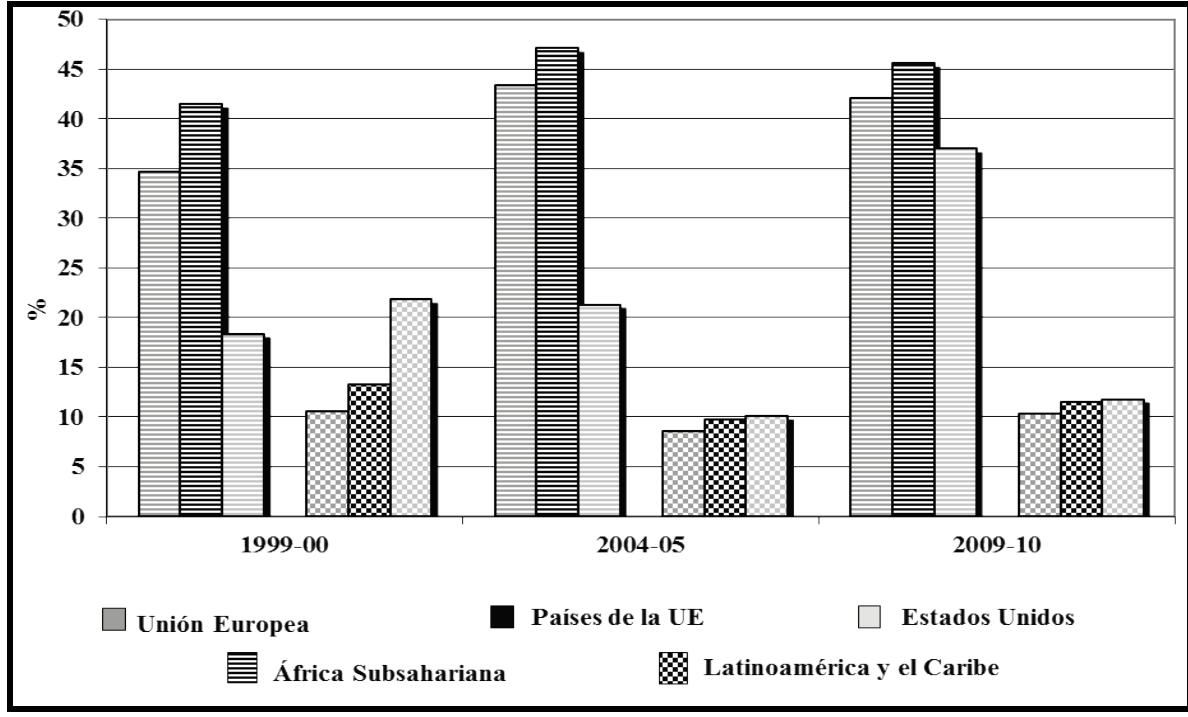

* Sin tener en cuenta la de destino especificado.

Fuente: Elaborado con datos del CAD (www.oecd.org/dac/stats).

Ahora bien, la apuesta por parte de la Unión Europea, tanto en los AAE suscritos al amparo del Convenio de Cotonú como en los AdA impulsados en América Latina, a favor del establecimiento de tratados de libre comercio, significa el casi total abandono de las concesiones no recíprocas y el establecimiento de un terreno de juego supuestamente equilibrado sobre la base de la reciprocidad en las preferencias otorgadas. $\mathrm{Y}$ era precisamente esa ausencia de reciprocidad la que conectaba positivamente una política comercial revestida de cierto carácter altruista con la cooperación para el desarrollo, llegando a convertir las preferencias comerciales no recíprocas en seña de identidad de su política de cooperación. Por eso, la exigencia de reciprocidad deja traslucir con toda clari- 
dad el predominio de los intereses económicos sobre cualquier apariencia de ayuda europea desinteresada.

Además, la orientación geográfica de los flujos de AOD de la refleja su uso como complemento de los tratados de libre comercio en la creciente competencia entre la Unión Europea y Estados Unidos en sus respectivas áreas de influencia tradicionales, si bien con diferentes tendencias. En efecto, como se ve en el Gráfico 2, EEUU destina aún una parte de su AOD a su tradicional área de influencia latinoamericana, si bien en una proporción cada vez más pequeña, y un porcentaje significativamente creciente hacia África Subsahariana. Mientras tanto la UE y sus países miembros mantienen a lo largo del tiempo el reparto de su AOD entre su área de influencia tradicional en África, así como en Latinoamérica y el Caribe. Se produce así una tendencia hacia un mayor equilibrio respecto a la capacidad de influencia de las potencias tradicionales sobre sus respectivas periferias por la vía de los flujos de AOD.

Por otra parte, ese libre comercio al que se subordina la cooperación se entiende en el sentido que le da la Organización Mundial de Comercio. Es decir, abarcando la liberalización del comercio internacional de servicios, además del de mercancías, y una mayor protección de la propiedad intelectual. Y la interpretación extensiva del término no se detiene ahí, sino que incluye también otros aspectos que, de momento, no son regulados por la OMC: las compras del sector publico, la política de competencia y la inversión extranjera.

En este sentido, la UE sigue el camino emprendido por Estados Unidos, que primero lanzó el proyecto del Acuerdo de Libre Comercio de las Américas $(\mathrm{ALCA})^{11}$, y luego lo transformó pragmáticamente en una suma de acuerdos bilaterales y subregionales. Todos ellos han seguido el modelo del Tratado de Libre Comercio de América del Norte (TLCAN, o NAFTA por sus siglas en inglés), negociado en paralelo a la Ronda Uruguay (1986-94) que dio lugar a la creación de la OMC en 1995. Por eso, el TLCAN abarca todo los temas incluidos en esta organización, es decir, comercio de bienes y servicios, protección de la propiedad intelectual, y algunos más, extendiendo el contenido del libre comercio mucho más allá de su significado tradicional.

Para ello la lógica utilizada es la siguiente: establecer que tal o cual cuestión está "relacionada con el comercio". Con esa simple coletilla se introdujo en la OMC algo en principio tan ajeno a la lógica de la liberalización o desregulación como la propiedad intelectual, a través del Acuerdo sobre Derechos de la Propiedad Intelectual relacionados con el Comercio (ADPIC). A pesar de que ya

${ }^{11}$ Formalmente las negociaciones del ALCA comenzaron en Miami en diciembre de 1994 para expandir el Tratado de Libre Comercio de América del Norte (TLCAN), al que ese mismo año se había adherido México. Pero ya en junio de 1990 el entonces presidente de EEUU, Bush padre, había propuesto la Iniciativa para las Américas, cuyo objetivo era lograr para 2000 una zona de libre comercio para el conjunto del continente, excepto Cuba. 
estaban regulados por la Organización Mundial de la Propiedad Intelectual (OMPI), la presión de las empresas multinacionales del Norte ${ }^{12}$ consiguió que pasaran a formar parte de la OMC, a fin de aprovechar la capacidad sancionadora de esta última para reforzar su protección. Ahora bien, no deja de resultar paradójico que el mismo ideario neoliberal que, encarnado en las reglas de la OMC, promueve la liberalización del comercio de bienes y servicios, ponga tanto empeño en aumentar la protección de la propiedad intelectual. Porque hay pocas cosas que tengan menos que ver con el mercado libre que las patentes: un monopolio, aunque sea temporal, otorgado por un estado. De hecho, no debería hablarse de derechos de propiedad intelectual, sino de monopolios artificiales sobre bienes intangibles, ya que esta denominación se ajusta mucho mejor a su verdadera función: privatizar el conocimiento, e incluso seres vivos, por mucho que se encubra bajo el eufemismo de formas de vida (Busaniche et al. 2007).

Después del éxito alcanzado con la incorporación del ADPIC, la lista de asuntos relacionados con el comercio que está pendiente de ser regulada por la OMC se alargó en su primera conferencia ministerial, celebrada en Singapur en 1996. Allí quedaron inscritas en la agenda para futuras negociaciones la política de competencia, las compras del sector público y la inversión extranjera ${ }^{13}$, luego conocidos en la jerga de la OMC como los temas de Singapur. El hecho de que en 2004 estos tres asuntos fueran retirados de las negociaciones de la Ronda de Doha, en un intento de evitar su paralización total tras el rotundo fracaso de la Conferencia ministerial de Cancún, contribuye a acentuar el interés de la UE y EEUU por seguir imponiéndolos en todos los tratados de libre comercio que suscriben.

Aunque en las reglas de la $\mathrm{OMC}$ las potencias económicas han logrado estirar el significado de la libertad comercial mucho más allá de lo razonable, no han dudado en incurrir en otra gran paradoja: la preconizada liberalización no alcanza a los movimientos internacionales de mano de obra no cualificados. En efecto, las migraciones laborales permanentes están excluidas de la liberaliza-

${ }^{12}$ El ADPIC es fruto directo de la labor de cabildeo (lobby) de las mismas empresas transnacionales que se benefician de él. En efecto, primero una docena de multinacionales estadounidenses de diversos sectores, aunque la mitad vinculadas a la farmacia y la biotecnología, constituyó un Comité de la Propiedad Intelectual (IPC por sus siglas en inglés) con el explícito objetivo de incluir ese asunto en la agenda de la Ronda Uruguay. Luego el IPC consiguió apoyos de empresas transnacionales europeas y japonesas para presionar a sus respectivos gobiernos. $\mathrm{Y}$ en 1988 una coalición de organizaciones empresariales de Europa, EEUU y Japón, en un hecho sin precedentes, se dirigió al entonces director del GATT y le propuso el borrador de lo que luego sería el ADPIC: "Basic Framework for GATT Provision on Intellectual Property". En ese documento figuran todos sus elementos esenciales, como una duración mínima de 20 años para las patentes y la extensión del campo de lo patentable a las formas de vida (CEO, 1999).

${ }^{13}$ Para no repetir la misma coletilla, los nombres oficiales de los grupos de trabajo que preparan esos asuntos para las negociaciones son: interacción entre comercio y política de competencia, transparencia de la contratación pública y relación entre comercio e inversiones. 
ción prevista en el Acuerdo sobre Comercio de Servicios (AGCS). Y en esto el TLCAN también sigue la pista de la OMC no contemplando su liberalización, a pesar de la enorme importancia que tiene el flujo migratorio entre México y Estados Unidos. Como en los demás asuntos, tanto el resto de TLC suscritos por EEUU como los AdA impulsados por la UE mantienen esta peculiar interpretación sobre el alcance del principio de liberalización de mercados.

Ahora bien, si se firman tratados de libre comercio no es para repetir lo que ya figura en la $\mathrm{OMC}$, sino para ampliarlo en determinados ámbitos. Por eso se suele decir que son acuerdos $O M C$-plus, ya que van más allá de lo comprometido en ella, profundizando la liberalización de lo que se pretende liberalizar y protegiendo más lo que se quiere proteger. Es decir, trazando las líneas de un terreno de juego global todavía más favorable para las empresas transnacionales al reforzar la liberalización de los flujos de mercancías y servicios, y de los movimientos de capitales, así como aumentar la protección de la propiedad intelectual y de los inversores extranjeros.

\section{JUSTIFICACIÓN DE LOS NUEVOS ACUERDOS E IMPLICACIONES PARA ÁFRICA Y AMÉRICA LATINA}

De acuerdo con lo visto, hay que entender el impulso de la Unión Europea a los tratados de libre comercio, llámense AAE o AdA, como una vía complementaria a la multilateral de la OMC para conseguir los mismos objetivos. Y estos no son otros que facilitar a las empresas transnacionales con base europea el acceso a los mercados africanos y latinoamericanos, tanto para abastecerse de materias primas y otros productos al menor coste como para colocar sus productos e inversiones en las mejores condiciones. Estos planteamientos van a salir además reforzados como respuesta a la grave crisis económica que sufren en la actualidad muchos países de la UE y la consiguiente reducción de los fondos de ayuda.

Con frecuencia tales objetivos prioritarios suelen ir acompañados de otros más loables, incluyendo el ámbito de la propia cooperación al desarrollo. De este modo, estos objetivos secundarios son promovidos en tanto en cuanto favorezcan, o al menos no obstaculicen, a los principales. Todo ello genera a menudo reticencias sobre el tipo de políticas de cooperación llevadas a cabo o sobre la coherencia entre éstas y el resto de las que componen los el amplio paquete de los Acuerdos de Asociación.

La Unión Europea justificó la profunda transformación de sus relaciones con los países ACP mediante un Libro Verde (Comisión Europea, 1997). El análisis de la UE partía de sostener la ineficacia del modelo de Lomé: por el incumplimiento de sus objetivos más ambiciosos, como la reducción de la pobreza o el logro de mayores niveles de desarrollo económico y social; pero también de otros más concretos y visibles a corto plazo, como el aumento del peso de sus 
productos en los mercados europeos, ya que el porcentaje de participación de las exportaciones de los países ACP en Europa había disminuido. Esto además contrastaba con la realidad de otras economías en desarrollo, en particular las asiáticas, que en ausencia de este trato preferencial habían conseguido aumentar el peso de sus exportaciones en los mercados europeos.

Después de considerar un fracaso el sistema de preferencias no recíprocas de Lomé, la UE subrayaba la incompatibilidad de los Acuerdos de Lomé con la normativa multilateral de la OMC, puesto que chocaba contra dos de sus principios básicos: reciprocidad y no-discriminación. La excepción al principio de reciprocidad, por la vía del artículo XXIV para el caso de acuerdos de integración regional, no era aplicable. Y aunque la violación de la cláusula de Nación Más Favorecida, representativa de la ausencia de discriminación, por parte del marco preferencial no recíproco de Lomé estaba en principio amparada por una excepción en virtud de la Cláusula de Habilitación, esta excepción exigía que tales ventajas fuesen aplicadas al conjunto de las economías en desarrollo y no únicamente a algunas de ellas (Gibb, 2000; McQueen, 1998).

Finalmente, la UE argumentaba que era necesario diferenciar entre los países ACP en función del nivel de desarrollo socioeconómico alcanzado y de su evolución durante las dos décadas anteriores. Sugería así reservar el tratamiento preferencial para los $\mathrm{PMA}^{14}$, considerados los más vulnerables, y no aplicarlo al resto de los ACP. Y además alegaba que esa era la forma de mejorar la eficiencia y competitividad de sus sistemas productivos y su capacidad de inserción en la economía mundial.

Ese diagnóstico del Libro Verde de la UE ha sido cuestionado y calificado de interesado, entre otras cosas porque de otro modo no se entiende la insistencia de los propios países afectados en mantener el modelo de Lomé y que, en su defecto, abogaban por la flexibilidad de los nuevos acuerdos en cuanto a temas sensibles y periodos de transición, de forma que permitieran un ajuste no traumático a la nueva situación (Hurt, 2003; Mold, 2007). De hecho, pese a las incompatibilidades señaladas, el modelo de Lomé continuó aplicándose durante las negociaciones que condujeron al Convenio de Cotonú y los AAE. Y, paradójicamente, la esencia de Lomé en términos de no-reciprocidad se seguirá aplicando para los PMA que lo deseen con la pretensión de favorecer sus condiciones de desarrollo (Faber \& Orbie, 2009).

${ }^{14}$ Es importante tener en cuenta los criterios a partir de los cuales se considera que unos países son menos desarrollados que otros. La clasificación de PMA obedece a los criterios de la UNCTAD utilizados también por la OMC. Pero no siempre coincide con la lista de países de bajo nivel de desarrollo del Banco Mundial, o con el índice de desarrollo humano (IDH) del PNUD. Así, países como Camerún, Congo, Costa de Marfil, Kenia y Nigeria alcanzan niveles de desarrollo en función del IDH inferiores a otros africanos considerados PMA. 
La estrategia Europa Global lanzada en 2006 por la Unión Europea explica con mayor claridad los motivos que se encuentran detrás de los AAE y AdA. Se trata esencialmente de ayudar a las empresas europeas a competir en una economía globalizada de manera que eso contribuya a la estrategia de crecimiento y empleo de la UE. De hecho, la patronal europea participó en su diseño y manifestó su acuerdo con el resultado (Olivet, 2008). Y enseguida propuso un conjunto muy detallado de características que debían tener los nuevos TLC negociados por la UE (Seillière, 2006; UNICE, 2006), que se corresponden perfectamente con los contenidos de los AAE y AdA que se van firmando.

Para lograr ese objetivo, con los acuerdos de asociación la UE se propone conseguir para las empresas europeas la apertura de los sectores de servicios y las compras gubernamentales; acceso seguro y barato a recursos como la energía, los metales y la chatarra, y a materias primas agrícolas; rebajas sustanciales o eliminación de aranceles de aduanas, barreras no arancelarias y todo tipo de subsidios; y mayor protección de la propiedad intelectual y las inversiones. En consonancia con la idea de que ahora más que nunca, Europa debe importar para poder exportar (Comisión Europea, 2006), en noviembre de 2008 la UE lanzó una iniciativa específica para cubrir sus necesidades de materias primas, de forma que se minimice el riesgo de futuros desabastecimientos de la industria europea (Amigos de la Tierra, 2008). Esto sería coherente con el proceso de reprimarización que se observa en un número creciente de países africanos y latinoamericanos, donde las potencias económicas tradicionales compiten ahora con las emergentes por sus abundantes recursos naturales.

Visto desde el punto de vista de los gobiernos de estos países que suscriben acuerdos de asociación con la UE, esto supone que se les sigue asignando mayormente el papel de suministradoras de materias primas para la industria europea, típico de la división internacional del trabajo tradicional, herencia de la era colonial. En la mayoría de los casos eso se ajusta a sus actuales relaciones comerciales con la Unión Europea, pero pretender perpetuarlas no parece la mejor vía para contribuir al desarrollo de estas economías. En efecto, salvo México, cuyos intercambios con la UE además de relativamente poco importantes $(6,8 \%$ de las exportaciones y $12 \%$ de la importaciones en 2008) son principalmente de productos manufacturados $(66 \%$ de la exportaciones y $81 \%$ de la importaciones), el resto sigue un patrón de comercio interindustrial, exportando mayoritariamente materias primas e importando sobre todo manufacturas (INTAL, 2010).

En promedio, para América Latina el comercio con la UE (exportaciones más importaciones) supone el 14,5\% del total en 2008, siendo Chile y el MERCOSUR los que más dependen de sus exportaciones a la UE, con algo más del $22 \%$ en ambos casos, y México el que menos. En cuanto a la concentración de las exportaciones en productos primarios destacan Chile, Colombia y Perú con 
cifras en torno al 90\% en 2008, seguidos por el Caribe y el MERCOSUR con cerca de las tres cuartas partes, y muy de lejos por Centroamérica donde apenas supone algo más de la mitad ${ }^{15}$ (INTAL, 2010). Además, la mayoría de las exportaciones de productos industriales latinoamericanos corresponde a manufacturas basadas en recursos naturales. En cambio, en todos los casos los principales productos importados desde la UE son manufacturados, rondando el $90 \%$ del total (Lara y Silva, 2009). Y el panorama del comercio entre los países ACP y la Unión Europea también es marcadamente interindustrial. En 2009, el $71,4 \%$ de las exportaciones de los países ACP a la UE fueron materias primas, de las cuales más de la mitad eran petróleo y minerales, mientras que el 77,7\% de las exportaciones de la UE a los países ACP correspondía a productos manufacturados (Comisión Europea, 2011).

Prolongar o incluso acentuar esta inserción subordinada y dependiente en la economía internacional firmando acuerdos que agudizan el modelo primario exportador o la producción manufacturera de bajo valor añadido y facilitan la entrada incondicional de las empresas europeas no conduce a nada que se parezca a una estrategia de desarrollo adecuada para estos países. De hecho, es muy probable que estos nuevos acuerdos contribuyan a la acentuada extraversión de la economía, la disminución significativa de los ingresos fiscales, así como al deterioro de las condiciones de vida de los pequeños productores agrícolas y la consolidación de la biopiratería, con graves consecuencias en el camino hacia el logro de la soberanía alimentaria (Amigos de la Tierra, 2008; Grain, 2008).

Respecto a los supuestos resultados positivos de una creciente entrada de inversión extranjera directa (IED), éstos deben ser relativizados en función de sus efectos sobre la creación de empleo, la calidad de éste, el efecto arrastre sobre la industria local, la transferencia tecnológica, etc. Y todos estos aspectos son directamente dependientes de la capacidad de los gobiernos para establecer requisitos de desempeño, es decir, condiciones que contribuyan a mejorar el carácter pro-desarrollo de dichas inversiones. No obstante, al igual que en el caso de los TLC latinoamericanos con EEUU, la tendencia aquí parece ser la contraria, de tal forma que el proyecto del fracasado Acuerdo Multilateral de Inversiones (AMI), vuelve ahora por la puerta de atrás como "AMI encubierto" de la mano de estos acuerdos regionales abiertos (Intermón-Oxfam, 2008; Bidaurratzaga, 2008).

Además, la mencionada estrategia Europa Global impone que cuando los acuerdos de asociación se hagan con socios que han firmado previamente TLC con competidores como mínimo se deben igualar las condiciones, que en todo

${ }^{15}$ Ahora bien, esto se debe a Panamá y, sobre todo, a Costa Rica, el mayor exportador de la región centroamericana a la UE, cuyas exportaciones son mayoritariamente de manufacturas (58,6\%) (Ocampo y Santamaría, 2009). 
caso irán más allá de lo ya acordado en la OMC. Y como las prioridades derivan de criterios económicos, más que de políticos, la lista de acuerdos a promover la encabezan la ASEAN, Corea del Sur y el MERCOSUR (Comisión Europea, 2006).

El que dos de las tres máximas prioridades futuras señaladas por la UE sean grupos de integración regional encaja muy bien con la idea de impulsar acuerdos con bloques subregionales, que eventualmente puedan confluir en un gran acuerdo de alcance regional. Ello también sería coherente con la firma de acuerdos con grupos regionales de Centroamérica, Caribe y alguna región africana. Interpretado en términos del llamado regionalismo abierto, esta estrategia es similar a la de Estados Unidos tras el fracaso del ALCA. Ambas pretenden atar formalmente las relaciones económicas con sus históricas áreas de influencia, en un contexto en el cual éstas se perciben además como parcialmente amenazadas por la competencia de algunas economías emergentes, no sólo en el ámbito del comercio y las inversiones, sino también en el de la ayuda proveniente de los "nuevos donantes". Esto es especialmente significativo en el caso de la creciente intensificación de las relaciones económicas y políticas con China, que tanta controversia está generando en el caso de África (Unceta y Bidaurratzaga, 2008).

Pero, más allá de la retórica, todo indica que la estrategia de la UE, al igual que en el caso de los EEUU, pasa con frecuencia más por dividir, para obtener mejores frutos de la acentuada asimetría y mayor debilidad de cada país de forma individual, que por unir. Ahí están los ejemplos de la mayor parte de los grupos regionales de África Subsahariana y América Latina, donde varias negociaciones que habían empezado con un grupo regional han derivado en acuerdos con países aislados o con subgrupos más pequeños. Y eso, lejos de promover y consolidar una integración regional que se integre en condiciones más favorables con el resto del mundo, contribuye a priorizar una integración hacia fuera que puede acabar desintegrando hacia dentro, mediante la distorsión y desestructuración de los procesos de integración regional en curso (Arroyo et al. 2009; Keet, 2007). En este sentido, no debe sorprender el impulso renovado de la cooperación Sur-Sur a partir de surgimiento de iniciativas regionales alternativas al modelo del regionalismo abierto, como en el caso Latinoaméricano ${ }^{16}$, o mediante el fortalecimiento de relaciones económicas y políticas con países emergentes (Morazán et al, 2011).

Las diferencias entre la retórica y la realidad no acaban ahí. Incluso en la parte blanda de los acuerdos de asociación resultan a menudo engañosas. Por

\footnotetext{
${ }^{16}$ Nos referimos, entre otros, a proyectos regionales como el ALBA-TCP (Alianza Bolivariana para los Pueblos de Nuestra América-Tratado de Comercio de los Pueblos), UNASUR (Unión de Naciones Suramericanas) o CELAC (Comunidad de Estados Latinoamericanos y Caribeños).
} 
ejemplo, en varios AdA la cooperación científica y tecnológica va integrada con privatización, patentes y otras formas de propiedad intelectual, mientras que la cooperación económica encubre mayor protección a la inversión. Y la parte dura de los TLC se impone sin ambigüedades, abarcando todos lo asuntos tratados por la OMC, incluidos los temas de Singapur, profundizando en su liberalización o protección según convenga. De este modo, el conjunto de los compromisos adquiridos en los acuerdos de asociación limita seriamente el margen de maniobra disponible por parte de los gobiernos que los suscriben para aplicar políticas nacionales o supranacionales de desarrollo. Y dadas las asimetrías productivas y comerciales de partida, poco pueden salir ganando estos países de igualar el terreno de juego mediante la concesión de preferencias recíprocas a una potencia económica como la Unión Europea, que además utiliza una política de dobles raseros con independencia de su discurso, como en el caso de la continuada protección a su sector agrícola. Es por eso fundamental atender a los argumentos de los que, junto a algunos gobiernos africanos y latinoamericanos, se oponen a estos TLC encubiertos desde los movimientos sociales, las organizaciones no gubernamentales y el mundo académico (Mira, 2007; Morales y Garza, 2009).

\section{CONCLUSIONES}

En los últimos años, la UE ha promovido de forma acelerada en África y América Latina unos acuerdos económicos que garanticen unas relaciones privilegiadas, básicamente en términos de comercio e inversiones, con su área tradicional de influencia, en el primer caso, y en competencia directa con el área de influencia de los EEUU, y siguiendo su estela en cuanto a tipos de acuerdo y contrapartes, en el segundo. Pese a la retórica de su discurso políticamente correcto, su presunta preocupación por la mejora de las condiciones de desarrollo y la especificidad de su modelo en cuanto a complementación de todo ello con fondos de cooperación al desarrollo, estos acuerdos generan grandes dudas y controversias sobre sus consecuencias socioeconómicas en los países de estas dos regiones.

$\mathrm{Su}$ insistencia en la reciprocidad comercial y en la inclusión en la agenda de negociación de temas controvertidos más allá de los acuerdos en la $\mathrm{OMC}$ no hará sino limitar las opciones de los gobiernos de la región para la elaboración de las políticas de desarrollo que consideren más convenientes. Los marcos de negociación subdivididos en pequeños grupos regionales o países individuales deterioran la capacidad de negociar sobre temas sensibles, condicionando el resultado de los acuerdos. Y, en contra del discurso oficial, dichos marcos de negociación distan de contribuir al fortalecimiento de los grupos regionales previamente conformados. 
Tal y como diferentes gobiernos, personas del mundo académico y movimientos sociales europeos, africanos y latinoamericanos han manifestado en los últimos años, estos acuerdos tienen serias limitaciones y contraindicaciones como instrumentos para la promoción del desarrollo en estas dos regiones, incluso yendo acompañados de proyectos y programas de ayuda al desarrollo.

En este sentido, el discurso oficial de la Comisión Europea a la hora de justificar los acuerdos en términos de apuesta por una estrategia que contribuya a la mejora de las condiciones de desarrollo en los países de estas dos grandes regiones no resulta consistente. El análisis de todo ello desde una perspectiva de coherencia de políticas es así fundamental, dado que no son pocas las voces cualificadas que interpretan que la propuesta interesada de regionalismo abierto de la UE de la mano de los AAE y los AdA no hará sino reducir el margen de maniobra de sus gobiernos y otros agentes sociales para el diseño e implementación de las políticas de desarrollo que mejor se adapten a cada uno de sus contextos y características específicas.

\section{REFERENCIAS BIBLIOGRÁFICAS}

AMIGOS DE LA TIERRA (2008): Europa Global: los verdaderos intereses de la estrategia comercial de la UE para someter a los pueblos a la tiranía del "libre comercio". Disponible en: www.foei.org

ARROYO, A.; RODRÍGUEZ, G. y CASTAÑEDA, N. (2009): La Unión Europea: ¿promotora de la integración regional en América Latina? Transnational Institute y Red Mexicana de Acción frente al Libre Comercio, abril. Disponible en: www.tni.org

BIDAURRATZAGA, E. (2008): "El nuevo modelo de relaciones Europa-África: El debate sobre los EPAs como instrumento de desarrollo", en Claves de la Economía Mundial, ICEX/ICEI, Madrid.

BUSANICHE, B. (ed.) (2007): Monopolios artificiales sobre bienes intangibles. El proceso de privatización de la vida y el conocimiento, Fundación Vía Libre, Córdoba, Argentina. Disponible en: www.vialibre.org.ar

CABALLEROS, R. (2008): "Centroamérica: los retos del Acueerdo de Asociación con la Unión Europea", Estudios y perspectivas n¹02, CEPAL, México.

CAD (2012): Base de datos del Comité de Ayuda al Desarrollo de la OCDE. Disponible en: www.oecd.org/dac/stats (fecha de consulta: 3-5-2012).

CEO (1999) "WTO Millennium Bug: TNC Control Over Global Trade Politics". Corporate Europe Observer $\mathrm{n}^{\circ} 4$, julio. Disponible en: www.corporateeurope.org 
COMISIÓN EUROPEA (1997): Green Paper on Relations between the European Union and the ACP Countries on the Eve of the $21^{\text {st }}$ Century, Challenges and Options for a New Partnership, Bruselas. Disponible en: http://europa.eu

COMISIÓN EUROPEA (2005): Una asociación reforzada entre la Unión Europea y América Latina, $\operatorname{COM(2005)} 636$ final. Disponible en: http://europa.eu

COMISIÓN EUROPEA (2006): Una Europa Global: competir en el mundo. Una contribución a la Estrategia de crecimiento y empleo de la UE, COM(2006) 567 final, 4 de octubre. Disponible en: http://europa.eu

COMISIÓN EUROPEA (2012): “Trade". Disponible en: http://ec.europa.eu/trade (fecha de consulta: 7-5-2011).

CONSEJO DE LA UE (2010): VI Cumbre UE-ALC. Declaración de Madrid, 18 de mayo. Disponible en: www.consilium.europa.eu

ECDPM, European Centre for Development Policy Management (2011): ACPEU Trade Website. Disponible en: www.acp-eu-trade.org (fecha de consulta: 25-11-2011).

EUROPEAN UNION (2006): Decision No 1/2006 of The ACP-EC Council Of Ministers. specifying the multiannual financial framework for the period 2008 to 2013 and modifying the revised ACP-EC Partnership Agreement. Official Journal of the European Union.

EUROSTAT (2010): "Africa-EU: economic indicators, trade and investment". Statistics in focus. N.59. http://ec.europa.eu/eurostat.

FABER, G. y ORBIE, J. (2009): “The EU's insistence on reciprocal trade with the ACP group: Economic interests in the driving seat?", en Gerrit Faber and Jan Orbie (eds), Beyond Market Access for Economic Development: EUAfrica relations in transition, Routledge, Londres.

GRAIN (2008): “Acuerdos con la Unión Europea ¿Firmar la renuncia a la soberanía nacional?", Documentos de análisis. Disponible en: www.grain.org

GRANADOS, J. (2009): “Los nudos gordianos de la negociación”, en Granados, Jaime, Eduardo Lizano y Fernando Ocampo (eds.): Un puente para el crecimiento: Oportunidades y desafíos del Acuerdo de Asociación entre Centroamérica y la Unión Europea, Banco Interamericano de Desarrollo, Academia de Centroamérica y Konrad-Adenauer-Stiftung, Washington.

GIBB, RICHARD (2000): "Post-Lomé, the European Union and the South", Third World Quarterly vol.21, n³, junio.

HURT, S. (2003): "Co-operation and coercion? The Cotonou Agreement between the European Union and ACP states and the end of the Lomé Convention", Third World Quarterly, vol.24, n¹, Londres.

INTAL (2010): Carta mensual, mayo. Disponible en: www.iadb.org/intal

KEET, D. (2007): Economic Partnership Agreements (EPAs): Responses to the EU Offensive against ACP Development Regions, Transnational Institute, Amsterdam. Disponible en: www.tni.org 
KUCHARZ, T. y VARGAS, M. (2010): "Tratados de Libre Comercio entre la Unión Europea y América Latina: una integración por y para el capital”, Boletín ODG, $\mathrm{n}^{\circ} 76$, abril. Disponible en: www.odg.cat

LARA, C. y SILVA, C. (2009): Las relaciones económicas entre Unión Europea - América Latina y sus impactos en los mercados laborales (1990-2007), Confederación Sindical de Trabajadores y Trabajadoras de las Américas, Santiago de Chile.

LIZANO, E.; OCAMPO, F. y ECHANDI, R. (2009): "Las oportunidades y los desafíos del Acuerdo de Asociación”, en Granados, Jaime, Eduardo Lizano y Fernando Ocampo (eds.): Un puente para el crecimiento: Oportunidades y desafíos del Acuerdo de Asociación entre Centroamérica y la Unión Europea, Banco Interamericano de Desarrollo, Academia de Centroamérica y Konrad-Adenauer-Stiftung, Washington.

MARIN, A. (2008): "Los Acuerdos de Asociación Económica (EPA) de la Unión Europea con África Subsahariana”. Documento de Trabajo, n¹9, Fundación Carolina - Fundación Alternativas, Madrid. Disponible en: www.falternativas.org

MCQUEEN, M. (1998): “ACP-EU trade cooperation after 2000. An assessment of reciprocal trade preferences", The Journal of Modern African Studies, vol. $36, \mathrm{n}^{\circ} 4$.

MIRANDA, S. y ECHANDI, R. (2009): “El diálogo político y la cooperación económica", en Granados, Jaime, Eduardo Lizano y Fernando Ocampo (eds.): Un puente para el crecimiento: Oportunidades y desafíos del Acuerdo de Asociación entre Centroamérica y la Unión Europea, Banco Interamericano de Desarrollo, Academia de Centroamérica y Konrad-Adenauer-Stiftung, Washington.

MIRA, E. (2007): El Acuerdo de Asociación Unión Europea - Centroamérica, un nuevo Tratado de Libre Comercio, CEICOM, El Salvador, diciembre. Disponible en: www.ceicom.org.sv

MOLD, A. (2007): “¿Retirada de último momento? Evaluaciones, opciones y alternativas a los Acuerdos de Asociación Económica (AEE)". Documento de Trabajo, n³3, Real Instituto Elcano. Disponible en: www.realinstitutoelcano.org

MORALES, H. y GARZA, J. (2009): El Acuerdo de Asociación entre la Unión Europea y Centroamérica, Movimiento Tzuk Kim-pop, Guatemala, diciembre. Disponible en: www.tzukkim-pop.org

MORAZÁN, P. et al (2011): Una nueva política de cooperación para el desarrollo de la Unión Europea con América Latina. Dirección General de Políticas Exteriores. Parlamento Europeo.

OCAMPO, F. y SANTAMARÍA, V. (2009): "Las relaciones comerciales y de inversión", en Granados, Jaime, Eduardo Lizano y Fernando Ocampo (eds.): Un puente para el crecimiento: Oportunidades y desafíos del Acuerdo de Asociación entre Centroamérica y la Unión Europea, Banco Interamericano 
de Desarrollo, Academia de Centroamérica y Konrad-Adenauer-Stiftung, Washington.

OCAMPO, F. y LIZANO, E. (2009): "El marco institucional de los países y de la región centroamericana", en Granados, Jaime, Eduardo Lizano y Fernando Ocampo (eds.): Un puente para el crecimiento: Oportunidades y desafíos del Acuerdo de Asociación entre Centroamérica y la Unión Europea, Banco Interamericano de Desarrollo, Academia de Centroamérica y Konrad-Adenauer-Stiftung, Washington.

OCAMPO, F.; LIZANO, E. y GRANADOS, J. (2009): "El futuro de las relaciones económicas entre Centroamérica y la Unión Europea", en Granados, Jaime, Eduardo Lizano y Fernando Ocampo (eds.): Un puente para el crecimiento: Oportunidades y desafíos del Acuerdo de Asociación entre Centroamérica y la Unión Europea, Banco Interamericano de Desarrollo, Academia de Centroamérica y Konrad-Adenauer-Stiftung, Washington.

OLIVET, C. (2008): "Europa Global: la estrategia europea de comercio e inversiones al servicio de las corporaciones", Pueblos, $n^{\circ} 34$, septiembre.

RAMIRO, P. y CARRIÓN, J. (2010): "Las relaciones Unión Europea - América Latina: de la cumbre oficial a la Cumbre de los Pueblos", Boletín de recursos de información del Centro de documentación de Hegoa, $n^{\circ} 23$, julio. Disponible en: www.hegoa.ehu.es

SEILLIĖRE, E.-A. (2006): "Delivering on Trade \& Competitiveness", Global Competition Conference, Bruselas, 13 de noviembre. Disponible en: www.businesseurope.eu

TRIBUNAL PERMANENTE DE LOS PUEBLOS (2010): La Unión Europea y las empresas trasnacionales en América Latina: politicas, instrumentos y actores complices de las violaciones de los derechos de los pueblos, Sentencia, Madrid, mayo. Disponible en: www.enlazandoalternativas.org

UNCETA, K. y BIDAURRATZAGA, E. (2008): "Las relaciones económicas chino-africanas y su incidencia sobre el patrón de desarrollo en el continente africano", Revista de Economía Mundial, n²0. Disponible en: http://www.sem-wes.org

UNICE (2006): UNICE Strategy on an EU Approach to Free Trade Agreements. Bruselas, 7 de diciembre. Disponible en: www.businesseurope.eu 